\title{
On Uniform Spaces with Invariant Nonstandard Hulls
}

\begin{abstract}
NADER VAKIL
Abstract: Let $\langle X, \Gamma\rangle$ be a uniform space with its uniformity generated by a set of pseudo-metrics $\Gamma$. Let the symbol " $\simeq$ " denote the usual infinitesimal relation on ${ }^{*} X$, and define a new infinitesimal relation " $\approx$ " by writing $x \approx y$ whenever ${ }^{*} \rho(x, p) \simeq{ }^{*} \rho(y, p)$ for each $\rho \in \Gamma$ and each $p \in X$. Let us call a uniform space $\langle X, \Gamma\rangle$ an $S$-space if the relations $\simeq$ and $\approx$ coincide on fin $\left({ }^{*} X\right)$. In [14], we showed that every S-space whose uniformity is generated by a single pseudometric has invariant nonstandard hulls. Here we extend that result to all uniform spaces and use it to explore further properties of S-spaces, which can now be recognized as uniform spaces that have invariant nonstandard hulls.
\end{abstract}

2010 Mathematics Subject Classification 54J05 (primary); 54D20, 54D60 (secondary)

Keywords: Nonstandard topology, Lindelöf spaces, Realcompactness

\section{Introduction}

The notation is as in the abstract above. The concept of an S-space arises in connection with the question of how to construct the nonstandard hull (Luxemburg [7]) of a uniform space $\langle X, \Gamma\rangle$ within the framework of Internal Set Theory (Nelson [8], Vakil [13]). In [14] we showed how this construction can be carried out for the class of S-spaces. It was also shown in [14] that the class of S-spaces whose uniformity is generated by a single pseudo-metric includes those that have invariant nonstandard hulls (INH). ${ }^{1}$

In Section 3 of the present paper, we extend our work in [14]. We introduce the internal notion of a pseudo-precompact (PSPC) uniform space and prove that a standard uniform

\footnotetext{
${ }^{1}$ Recall that $\langle X, \Gamma\rangle$ has $I N H$ if fin $\left({ }^{*} X\right)=\operatorname{pns}\left({ }^{*} X\right)$. This notion was introduced and studied by C. W. Henson and L. C. Moore in [5] and [6]. In the context of locally convex spaces, uniform space with $I N H$ occur as HM-spaces, of which nuclear and Schwarz spaces are examples. For a discussion of HM-spaces, see Stroyan and Luxemberg [12, page 312] and its references to the works of C. W. Henson and L. C. Moore on this subject.
} 
space is an S-space if and only if it is PSPC. Moreover, we show that the S-space property and the INH property are equivalent for all standard uniform spaces. Thus, in the notion of a PSPC space we provide a new internal characterization of the familiar INH property. The class of PSPC spaces extends the class of precompact uniform spaces, and Section 3 contains a discussion of two useful examples of non-precompact PSPC spaces. One example obtains by equipping a completely regular space $\langle X, \tau\rangle$ that admits at least one unbounded $f \in C(X)$ with the weakest uniformity $\mathcal{U}_{c}$ with respect to which every $f \in C(X)$ is uniformly continuous (Example 3.8). Another example is the uniform space $\left\langle X, \mathcal{U}_{\phi}\right\rangle$, where $\mathcal{U}_{\phi}$ is the finest compatible uniform structure on $\langle X, \tau\rangle$. This is proved in Theorem 3.11, which requires the additional condition that the space $\langle X, \tau\rangle$ be devoid of discrete closed subspaces that have measurable cardinality.

Applications are presented in Section 4, which begins with the observation that the notion of a PSPC space provides a link between the theory of uniform spaces that have invariant nonstandard hulls and the theory of uniform spaces that have a unique structure. Through this link, we obtain two sufficient conditions for a uniform space to have invariant nonstandard hulls. Next we present a nonstandard proof of the fact that the uniform space $\left\langle X, \mathcal{U}_{c}\right\rangle$ is complete if $\langle X, \tau\rangle$ is Lindelöf, thus providing a nonstandard proof of the well-known result that a completely regular space that is Lindelöf is realcompact. This leads to another application of the PSPC property through Theorem 4.7, which states that if a topological space $\langle X, \tau\rangle$ is $T_{0}$, regular and Lindelöf then a subset $S$ of $X$ is relatively compact if and only if $f[S]$ is bounded for each $f \in C(X)$. Our simple proof uses only the observation that if a complete uniform space $\langle X, \mathcal{U}\rangle$ is PSPC then a subset $S$ of $X$ is relatively compact if and only if it is bounded.

\section{Preliminaries}

In this section we fix some notation and terminology concerning uniform structures and their nonstandard theory. We work with pairs $\langle X, \Lambda\rangle$, where $X$ is an infinite set and $\Lambda$ is a set of pseudo-metrics on $X$. The uniformity generated by $\Lambda$ will be denoted by $\mathcal{U}_{\Lambda}$. The pair $\left\langle X, \mathcal{U}_{\Lambda}\right\rangle$ as well as the pair $\langle X, \Lambda\rangle$ will be referred to as the uniform space generated by $\Lambda$. We also recall that if we begin with a uniformity $\mathcal{U}$ (rather than a set of pseudo-metrics $\Lambda$ ) on $X$ then $\mathcal{U}$ may be regarded as the uniformity generated by the set $\Lambda_{u}$ of all the pseudo-metrics on $X$ that are uniformly continuous on the space $X \times X$ equipped with the uniform structure $\mathcal{U} \times \mathcal{U}$. The set $\Lambda_{u}$ is called the gauge of $\mathcal{U}$ and we have $\mathcal{U}=\mathcal{U}_{\Lambda_{u}}$. If $\bar{\Lambda}$ denotes the set of all the uniformly continuous 
pseudo-metrics on $\langle X, \Lambda\rangle$ then $\Lambda \subseteq \bar{\Lambda}$. The set $\bar{\Lambda}$ is the gauge of $\mathcal{U}_{\Lambda}$ and we have $\mathcal{U}_{\Lambda}=\mathcal{U}_{\bar{\Lambda}}$. A subset $G$ of $X$ is called $\Lambda$-bounded if $\sup _{x, y \in G} \rho(x, y)<\infty$ for all $\rho \in \Lambda$. We refer to $G$ as $\mathcal{U}_{\Lambda}$-bounded in case it is $\bar{\Lambda}$-bounded. For the basic theory of uniform spaces, the reader may refer to Bourbaki [1], Page [10] or Willard [15].

As we mentioned earlier, the subject of this paper was inspired by the question of representation of nonstandard hulls within Internal Set Theory (IST). It is thus natural that we use IST (as outlined in Nelson [8] and expounded in Vakil [13]) as our nonstandard framework. Nevertheless, all arguments in this paper would be trivial to move over into the other foundational frameworks of NSA.

We assume that the reader is familiar with external and internal formulas (see Vakil [13, page 20]), the transfer axiom [13, page 35], the idealization axiom [13, page 71] and the standardization axiom [13, page 78]. We recall that if $S$ is a standard set and $\phi$ is a formula (external or internal) then, by the standardization axiom, $S$ has a unique standard subset $T$ whose standard elements satisfy $\phi$. We shall have occasions to use this fact in the work ahead. ${ }^{2}$ Given a class $A$ by ${ }^{\sigma} A$ we denote the class of all the standard members of $A$. Let $X$ be a standard infinite set and let $\mathcal{F}$ be a standard filter on $X$. We recall that the monad of $\mathcal{F}$ is defined to be the class $\mu(\mathcal{F})=\bigcap_{F \in \sigma_{\mathcal{F}}} F$. Moreover, by the idealization axiom there always exists an element $F \in \mathcal{F}$ such that $F \subseteq \mu(\mathcal{F})$. The basic concepts and results concerning the nonstandard theory of uniform spaces are reviewed next.

2.1 Definition Let $\Lambda$ be a standard family of pseudo-metrics on an infinite standard set $X$. Then we write:

(a) $\operatorname{fin}_{\Lambda}(X)=\left\{x \in X:\left(\forall \rho \in{ }^{\sigma} \Lambda\right)\left(\forall p \in{ }^{\sigma} X\right)\left(\exists M \in{ }^{\sigma} \mathbb{R}^{+}\right)[\rho(x, p)<M]\right\}$,

(b) $\operatorname{pns}_{\Lambda}(X)=\left\{x \in X:\left(\forall \epsilon \in{ }^{\sigma} \mathbb{R}^{+}\right)\left(\forall \rho \in{ }^{\sigma} \Lambda\right)\left(\exists p \in{ }^{\sigma} X\right)[\rho(x, p)<\epsilon]\right\}$,

(c) $\operatorname{ns}_{\Lambda}(X)=\left\{x \in X:\left(\exists p \in{ }^{\sigma} X\right)\left(\forall \epsilon \in{ }^{\sigma} \mathbb{R}^{+}\right)\left(\forall \rho \in{ }^{\sigma} \Lambda\right)[\rho(x, p)<\epsilon]\right\}$, and

(d) $\mu_{\Lambda}(x)=\left\{y \in X:\left(\forall \epsilon \in{ }^{\sigma} \mathbb{R}^{+}\right)\left(\forall \rho \in{ }^{\sigma} \Lambda\right)[\rho(x, y)<\epsilon]\right\}$, where $x \in X$.

(e) $\mu\left(\mathcal{U}_{\Lambda}\right)=\left\{\langle x, y\rangle \in X \times X:\left(\forall \epsilon \in{ }^{\sigma} \mathbb{R}^{+}\right)\left(\forall \rho \in{ }^{\sigma} \Lambda\right)[\rho(x, y)<\epsilon]\right\}$.

The members of the classes (a)-(c) are called, respectively, finite, pre-nearstandard, and nearstandard. It is evident that

$$
{ }^{\sigma} X \subseteq \mathrm{ns}_{\Lambda}(X) \subseteq \operatorname{pns}_{\Lambda}(X) \subseteq \operatorname{fin}_{\Lambda}(X) .
$$

Recall that the space $\langle X, \Lambda\rangle$ is compact if and only if $X=\mathrm{ns}_{\Lambda}(X)$, and that $\langle X, \Lambda\rangle$ is complete if and only if $\mathrm{ns}_{\Lambda}(X)=\operatorname{pns}_{\Lambda}(X)$ (see Luxemburg [7, Theorem 3.14.1, page

\footnotetext{
${ }^{2}$ This unique $T$ may be denoted by $*\{x \in S: \phi(x)\}$, or by ${ }^{s}\{x \in S: \phi(x)\}$.
} 
78]). ${ }^{3}$ The class $\mu_{\Lambda}(x)$ is called the monad of $x$. Note that the class $\mu\left(\mathcal{U}_{\Lambda}\right)$ is indeed the monad of the filter $\mathcal{U}_{\Lambda}$. We usually write $x \simeq_{\Lambda} y$ instead of $\langle x, y\rangle \in \mu\left(\mathcal{U}_{\Lambda}\right)$. We also note that $x \in \mu_{\Lambda}(y)$ if and only if $x \simeq_{\Lambda} y$, and

$$
x \simeq_{\Lambda} y \quad \text { if and only if } \quad \rho(x, y) \simeq 0 \text { for all } \rho \in{ }^{\sigma} \Lambda .
$$

We will use the notations $\mu\left(\mathcal{U}_{\Lambda}\right)$ and $\simeq_{\Lambda}$ interchangeably. For a standard topological space $\langle X, \tau\rangle$ and a $c \in{ }^{\sigma} X$, the monad of $c$, denoted $\mu_{\tau}(c)$, is defined to be the class of all $x \in X$ that belong to every standard $G \in \tau$ with $c \in G$. The class of nearstandard points of $\langle X, \tau\rangle$ is defined to be the class

$$
\mathrm{ns}_{\tau}(X)=\bigcup_{c \in \sigma^{\sigma}} \mu_{\tau}(c)
$$

Notice that $\mathrm{ns}_{\tau}(X)=\mathrm{ns}_{\Lambda}(X)$ in case $\tau$ is a uniformizable topology induced by a set of pseudo-metrics $\Lambda$. As to the the real line $\mathbb{R}$, we use the notation $\simeq$ for the usual infinitesimal relation in $\mathbb{R}$, and we call a real number $x$ limited if $|x|<M$ for some standard $M \in \mathbb{R}$. We call $x$ unlimited and write $|x| \simeq \infty$ if it is not limited. If $x \in \mathbb{R}$, then ${ }^{\circ} x$ is the unique element of ${ }^{\sigma} \mathbb{R} \cup\{-\infty, \infty\}$ such that $x \simeq{ }^{\circ} x$. This is called the standard part of $x$, and it is a standard real number if $x$ is limited, and it is $\pm \infty$ if $x$ is unlimited. The next theorem is well known, and we omit the proof.

2.2 Theorem Let $\langle X, \Lambda\rangle$ be a standard uniform space. Then we have:

(i) If $x \in \operatorname{fin}_{\Lambda}(X)$ and $\mathcal{F}(x)$ is the standard subset of $\mathcal{P}(X)$ whose standard elements contain $x$ then $\mathcal{F}(x)$ is a $\Lambda$-bounded ultrafilter on $X$.

(ii) A standard filter $\mathcal{F}$ on $X$ is $\Lambda$-bounded if and only if $\mu(\mathcal{F}) \subseteq \operatorname{fin}_{\Lambda}(X)$.

\section{Pseudo-Precompact Spaces}

The concept of a pseudo-precompact space introduced in this section is an internal notion equivalent (for standard sets) to the external notion of an S-space (see the abstract above). It is also equivalent (for standard sets) to the familiar notion of uniform spaces that have INH. This latter equivalence obtains through Theorem 3.7, which is the main theorem of this section. Interesting connections between the INH property and other properties of uniform spaces are revealed through Theorem 3.7. This and other applications of the notion of a pseudo-precompact space will be discussed in Section 4. In this section, we define the concept and provide some basic information about it. We begin by recalling the notion of a bounded filter in a uniform space.

\footnotetext{
${ }^{3} \mathrm{~A}$ proof of this theorem in the context of a metric space can be found in Vakil [13, page 412].
} 
3.1 Definition (Bounded Filters) Let $\langle X, \mathcal{U}\rangle$ be a uniform space, and let $\mathcal{F}$ be a filter on $X$. We call $\mathcal{F}$ bounded (precompact) if it has an element that is $\mathcal{U}$-bounded $(\mathcal{U}$-precompact).

3.2 Definition (Coinciding on Filters) Let $X$ be an infinite set, let $\mathcal{F}$ be a filter on $X$, and let $\mathcal{U}$ and $\mathcal{V}$ be two uniform structures on $X$. We say that $\mathcal{U}$ and $\mathcal{V}$ coincide on $\mathcal{F}$ if there is a $G \in \mathcal{F}$ such that

$$
\{(G \times G) \cap U: U \in \mathcal{U}\}=\{(G \times G) \cap V: V \in \mathcal{V}\} .
$$

3.3 Definition (Anchored Dual of a Uniformity) Let $\langle X, \Lambda\rangle$ be a uniform space. For each $\rho \in \Lambda$ and $p \in X$, let $\rho_{p}(x, y)=|\rho(x, p)-\rho(y, p)|$ for all $x, y \in X$. If $\Lambda^{\prime}=\left\{\rho_{p}: \rho \in \Lambda, p \in X\right\}$ then we call the uniformity $\mathcal{U}_{\Lambda^{\prime}}$ the anchored dual of the uniformity $\mathcal{U}_{\Lambda}$. In case we begin with a uniformity $\mathcal{U}$ on $X$, the anchored dual of $\mathcal{U}$ is the uniformity $\mathcal{U}^{\prime}$ that is generated by the set $\Lambda_{u}^{\prime}=\left\{\rho_{p}: \rho \in \Lambda_{u}, p \in X\right\}$, where $\Lambda_{u}$ is the gauge of $\mathcal{U}$.

Notice that when $\langle X, \Lambda\rangle$ is a standard uniform space we have

$$
x \simeq_{\Lambda^{\prime}} y \text { if and only if } \rho(x, p) \simeq \rho(y, p) \text { for all } \rho \in{ }^{\sigma} \Lambda, p \in{ }^{\sigma} X .
$$

It is not difficult to see that a uniformity $\mathcal{U}$ on $X$ and its anchored dual $\mathcal{U}^{\prime}$ induce the same topology on $X$. Moreover, it follows easily from the triangle inequality that in general $\mathcal{U}^{\prime} \subseteq \mathcal{U}$. The equality holds in case $\langle X, \mathcal{U}\rangle$ is precompact, but we are interested in the following weaker equality condition.

3.4 Definition (Pseudo-Precompactness) We call a uniform space $\langle X, \Lambda\rangle$ pseudoprecompact if $\mathcal{U}_{\Lambda}$ and its anchored dual $\mathcal{U}_{\Lambda^{\prime}}$ coincide on all $\Lambda$-bounded ultrafilters on $X$. We shall abbreviate the term "pseudo-precompact" by PSPC.

In Theorem 3.7 below, we prove that a standard uniform space is PSPC if and only of it has $I N H$. The following two results pave the way for that theorem.

3.5 Theorem Let the notation be as in Definition 3.4. If $\langle X, \Lambda\rangle$ is standard then the two infinitesimal relations $\simeq_{\Lambda}$ and $\simeq_{\Lambda^{\prime}}$ coincide on $\operatorname{pns}_{\Lambda}(X)$.

Proof The inclusion $\mu\left(\mathcal{U}_{\Lambda}\right) \subseteq \mu\left(\mathcal{U}_{\Lambda^{\prime}}\right)$ is an immediate consequence of the triangle inequality. For the reverse inclusion, fix $x, y \in \operatorname{pns}_{\Lambda}(X)$ with $\rho(x, p) \simeq \rho(y, p)$ for each standard $\rho \in \Lambda$ and each standard $p \in X$. Fix a standard $\epsilon \in \mathbb{R}^{+}$. Since $x \in \operatorname{pns}_{\Lambda}(X)$, 
there is a standard point $p \in X$ such that $\rho(x, p)<\epsilon / 3$. Since $\rho(x, p) \simeq \rho(y, p)$, we have $\rho(y, p)<\epsilon / 2$. We can thus write

$$
\rho(x, y) \leq \rho(x, p)+\rho(y, p)<\epsilon,
$$

and the proof is complete.

3.6 Lemma If $\langle X, \Lambda\rangle$ is a standard PSPC space then $\operatorname{fin}_{\Lambda}(X) \subseteq \operatorname{pns}_{\Lambda}(X)$.

Proof Assume that $\langle X, \Lambda\rangle$ is a standard PSPC space. Fix $x \in \operatorname{fin}_{\Lambda}(X), \rho \in \Lambda$ and $\epsilon \in \mathbb{R}^{+}$, where $\rho$ and $\epsilon$ are standard. Let $\mathcal{F}(x)$ be the unique standard subset of $\mathcal{P}(X)$ whose standard elements contain $x$. By Theorem 2.2, $\mathcal{F}(x)$ is a bounded ultrafilter. Hence, by hypothesis and the transfer axiom, there is a standard $B \in \mathcal{F}(x)$ on which $\mathcal{U}_{\Lambda}$ and $\mathcal{U}_{\Lambda^{\prime}}$ are identical. Therefore there exist a standard $\delta \in \mathbb{R}^{+}$and a standard finite subset $F=\left\{p_{1}, \ldots, p_{n}\right\}$ of $X$ such that the set

$$
U=\left\{\langle u, v\rangle \in B^{2}: \max _{i}\left|\rho\left(u, p_{i}\right)-\rho\left(v, p_{i}\right)\right|<\delta\right\} .
$$

is contained in the set $V=\left\{\langle u, v\rangle \in B^{2}: \rho(u, v)<\epsilon\right\}$. Notice that $U$ and $V$ are standard, and that $U[x] \subseteq V[x]$. Now let $a_{i}={ }^{\circ} \rho\left(x, p_{i}\right)$, and let

$$
A=\left\{v \in B: \max _{i}\left|a_{i}-\rho\left(v, p_{i}\right)\right|<\frac{\delta}{2}\right\} .
$$

Clearly, $A$ is a standard subset of $X$ with $x \in A$. From the latter, it follows that $A \neq \emptyset$. Pick a standard point $q \in A$. Since $A \subseteq U[x] \subseteq V[x]$, we have $\rho(x, q)<\epsilon$, which yields $x \in \operatorname{pns}_{\Lambda}(X)$ by Definition 2.1(b). The proof is complete.

We are now ready for the main theorem of this paper.

3.7 Theorem Let $\langle X, \Lambda\rangle$ be a standard uniform space. The following three conditions are equivalent.

(i) The space $\langle X, \Lambda\rangle$ is pseudo-precompact.

(ii) The space $\langle X, \Lambda\rangle$ has invariant nonstandard hulls. That is, we have

$$
\operatorname{fin}_{\Lambda}(X)=\operatorname{pns}_{\Lambda}(X) .
$$

(iii) The space $\langle X, \Lambda\rangle$ is an S-space. That is, the relations $\simeq_{\Lambda}$ and $\simeq_{\Lambda^{\prime}}$ coincide on $\operatorname{fin}_{\Lambda}(X)$. 
Proof Since the inclusion $\operatorname{pns}_{\Lambda}(X) \subseteq \operatorname{fin}_{\Lambda}(X)$ holds in general, the implication $[(\mathrm{i}) \rightarrow$ (ii)] is an immediate consequence of Lemma 3.6. The implication [(ii) $\rightarrow$ (iii)] is trivial in light of Theorem 3.5. To prove [(iii) $\rightarrow$ (i)], fix a standard $\Lambda$-bounded ultrafilter $\mathcal{F}$ on $X$ and pick any standard $\rho \in \Lambda$. Then by Definition 3.2 and the transfer axiom there is a standard $G \in \mathcal{F}$ and a standard $M \in \mathbb{R}^{+}$with $\rho(x, y)<M$ for all $x, y \in G$. This implies that $G \subseteq \operatorname{fin}_{\Lambda}(X)$. Hence, by (iii), the relations $\simeq_{\Lambda}$ and $\simeq_{\Lambda^{\prime}}$ coincide on $G$. Since $\simeq_{\Lambda}$ is the monad of $\mathcal{U}_{\Lambda}$ and $\simeq_{\Lambda^{\prime}}$ is the monad of $\mathcal{U}_{\Lambda^{\prime}}$, it follows that the uniformities $\mathcal{U}_{\Lambda}$ and $\mathcal{U}_{\Lambda^{\prime}}$ are identical on $G$. Apply the transfer axiom to see that each bounded ultrafilter has an element on which $\mathcal{U}_{\Lambda}$ and $\mathcal{U}_{\Lambda^{\prime}}$ are identical. The proof of the theorem is now complete.

Recall that a standard uniform space is precompact if and only if $\operatorname{pns}(X)=X$. Hence, by Theorem 3.7 each precompact uniform space is PSPC. But the converse does not hold. The simplest example is the Euclidean space $\mathbb{R}^{n}$, where the uniformity is generated by its usual norm. Here is another example.

3.8 Example Any completely regular space that admits at least one unbounded continuous function has a compatible uniformity that is PSPC without being precompact.

Proof Let $\langle X, \tau\rangle$ be completely regular that admits at least one unbounded real-valued continuous function. Let $C(X)$ be the set of all the real-valued continuous functions on $X$, and for each $f \in C(X)$, let $\rho_{f}(x, y)=\mid f(x)-f(y)$. It is well known that the uniform structure on $X$ that is generated by the set $\Lambda_{c}=\left\{\rho_{f}: f \in C(X)\right\}$ is compatible with $\tau$. To see that the space $\left\langle X, \Lambda_{c}\right\rangle$ is pseudo-precompact, assume that it is standard, and verify that

$$
\operatorname{fin}_{\Lambda_{c}}(X)=\left\{x \in X: f(x) \text { is limited for all } f \in{ }^{\sigma} C(X)\right\} .
$$

By Theorem 3.7, we are done once we show that $\operatorname{fin}_{\Lambda_{c}}(X) \subseteq \operatorname{pns}_{\Lambda_{c}}(X)$. Fix $x \in$ $\operatorname{fin}_{\Lambda_{c}}(X), f \in{ }^{\sigma} C(X)$, and $\epsilon \in{ }^{\sigma} \mathbb{R}^{+}$. Let $a={ }^{\circ} f(x)$, and let

$$
A=\{u \in X:|f(u)-a|<\epsilon / 2\} .
$$

Then $A$ is a standard set and it is not empty because it contains $x$. So $A$ also contains a standard element $p$. Hence $\rho_{f}(x, p)=|f(x)-f(p)|<\epsilon$ for some standard $p \in X$, which completes the proof that $x \in \operatorname{pns}_{\Lambda_{c}}(X)$. We have thus shown that $\left\langle X, \Lambda_{c}\right\rangle$ is pseudo-precompact. By transfer, there is a standard $f \in C(X)$ that is not bounded. Hence there is an $x \in X$ with $f(x)$ unlimited. Hence, by 3.8.1 $x \notin \operatorname{fin}_{\Lambda_{c}}(X)$. Therefore $x \notin \operatorname{pns}_{\Lambda_{c}}(X)$. Since $\left\langle X, \Lambda_{c}\right\rangle$ is precompact if and only if $X=\operatorname{pns}_{\Lambda_{c}}(X)$, it follows that $\left\langle X, \Lambda_{c}\right\rangle$ is not precompact. 
Let $\langle X, \tau\rangle$ be a completely regular space, let $\mathcal{U}_{\phi}$ be the finest uniform structure on $X$ that is compatible with $\tau$, and let $\mathcal{U}_{c}$ be the uniform structure on $X$ that is generated by the set of pseudo-metrics $\Lambda_{c}$ described in Example 3.8. Part (i) of Theorem 3.11 provides another example of a non-precompact PSPC space. We need the next theorem, which is due to C. Ward Henson [5].

3.9 Theorem (Henson) Let $\langle X, \tau\rangle$ be a standard completely regular space. The following two conditions hold.

(i) For each $x, y \in \operatorname{pns}_{\phi}(X)$, we have $x \simeq_{\phi} y$ if and only if $x \simeq_{c} y$.

(ii) We have $\operatorname{pns}_{\phi}(X)=\mathrm{fin}_{c}(X)$ provided that $X$ has no closed discrete subspace of measurable cardinality.

Proof See [5, Theorem 2, page 165].

The notion of boundedness in an arbitrary topological space may be defined as follows. We need this notion in the next theorem and in Theorem 4.7.

3.10 Definition (Topologically Bounded Sets) Let $\langle X, \tau\rangle$ be a topological space, let $S$ be a subset of $X$, and let $\mathcal{F}$ be a filter on $X$. We call $S$ topologically bounded if its image $f[S]$ under each $f \in C(X)$ is bounded. We call $\mathcal{F}$ topologically bounded if it has a topologically bounded element.

3.11 Theorem Let $\langle X, \tau\rangle$ be a completely regular space that admits at least one unbounded continuous function. If $X$ has no closed discrete subspace of measurable cardinality then we have:

(i) The uniform space $\left\langle X, \mathcal{U}_{\phi}\right\rangle$ is a non-precompact PSPC space.

(ii) The uniformities $\mathcal{U}_{c}$ and $\mathcal{U}_{\phi}$ coincide on topologically bounded ultrafilters.

Proof Assume the data are standard. For (i), by Theorem 3.7, we need only show that $\operatorname{fin}_{\phi}(X) \subseteq \operatorname{pns}_{\phi}(X)$. Since $\mathcal{U}_{c} \subseteq \mathcal{U}_{\phi}$, we have $\operatorname{fin}_{\phi}(X) \subseteq$ fin $_{c}(X)$. Now use part (ii) of Theorem 3.9 to complete the proof of (i). Condition (ii) is equivalent to stating that $x \simeq_{\phi} y$ if and only if $x \simeq_{c} y$ for each $x, y \in \operatorname{fin}_{c}(X)$. By part (i) of Theorem 3.9, we have $x \simeq_{\phi} y$ if and only if $x \simeq_{c} y$ for each $x, y$ in pns $\phi_{\phi}(X)$, which equals $\operatorname{fin}_{c}(X)$ by part (ii) of Theorem 3.9. The proof of (ii) is complete.

Subspaces, projective limits, Cartesian products and uniformly continuous images of PSPC spaces are PSPC spaces. Most of these have straightforward proofs. Here is a precise statement and a proof of the last result in the list. 
3.12 Theorem Let $\langle X, \Lambda\rangle$ and $\langle Y, \Gamma\rangle$ be uniform spaces, and let $f: X \rightarrow Y$ be a uniformly continuous surjection such that the filter $f^{-1}(\mathcal{G})$ is $\Lambda$-bounded for each $\Gamma$-bounded ultrafilter $\mathcal{G}$ on $Y$. If $\langle X, \Lambda\rangle$ is PSPC then $Y$ is also PSPC.

Proof Assume that the data are standard. Fix $y \in \operatorname{fin}_{\Gamma}(Y), \gamma \in{ }^{\sigma} \Gamma$, and $\epsilon \in{ }^{\sigma} \mathbb{R}^{+}$. Since $f$ is uniformly continuous, by the transfer axiom there is an $F \in{ }^{\sigma} \mathcal{F} \operatorname{in}(\Lambda)$ and a $\delta \in{ }^{\sigma} \mathbb{R}^{+}$such that

$$
\left(\forall x_{1}, x_{2} \in X\right)\left[\max _{d \in F} d\left(x_{1}, x_{2}\right)<\delta \rightarrow \gamma\left(f\left(x_{1}\right), f\left(x_{2}\right)\right)<\epsilon\right] .
$$

Now fix an $x \in X$ with $y=f(x)$. Let $\mathcal{F}(x)$ be the standard ultrafilter whose standard elements contain $x$. Then the set $\mathcal{G}=f(\mathcal{F}(x))$ is an ultrafilter in $Y$ whose standard elements contain $y$. Since $y \in \operatorname{fin}_{\Gamma}(Y)$, the filter $\mathcal{G}$ is $\Gamma$-bounded by Theorem 2.2. By hypothesis, $\left.f^{-1}(\mathcal{G})\right)$ is $\Lambda$-bounded, and we have $\mu(\mathcal{F}(x)) \subseteq \mu\left(f^{-1}(\mathcal{G})\right) \subseteq \operatorname{fin}_{\Lambda}(X)$ by Theorem 2.2. This implies that $x$ is finite, and hence pre-nearstandard because $X$ is PSPC. Therefore there is a standard $p \in X$ with $\max _{d \in F} d(x, p)<\delta$. So, by formula 3.12.1, $\gamma(f(x), f(p))<\epsilon$. Since $f(p)$ is standard, this proves that $y=f(x) \in \operatorname{pns}_{\Gamma}(Y)$, and the proof is complete.

\section{Some Applications}

Let $\langle X, \tau\rangle$ be a Hausdorff completely regular space, and let $\mathcal{U}_{\tau}$ be a compatible uniform structure on $X$. Since $\mathcal{U}_{\tau}$ and its anchored dual $\mathcal{U}_{\tau}^{\prime}$ are both compatible with $\tau$, if $\langle X, \tau\rangle$ admits only one compatible uniform structure, then the uniform space $\left\langle X, \mathcal{U}_{\tau}\right\rangle$ is PSPC. The uniqueness of uniform structure was studied by several authors in the 1950s (see eg Doss [3], Dickinson [2] and newns [9]). The internal notion of PSPC provides a link between this uniqueness theory and the theory of uniform spaces with INH through Theorem 3.7. This is illustrated in the next two theorems. The first is in terms of functionally separated pairs of subsets. By a functionally separated pair of subsets of a topological space $X$ we mean a pair $A, B$ for which there is a real-valued continuous function such that $f[A]=\{0\}$ and $f[B]=\{1\}$.

4.1 Theorem Let $\langle X, \tau\rangle$ be a Hausdorff completely regular space. If for each functionally separated pair of closed sets at least one is compact then each compatible uniformity on $X$ is a PSPC space. 
Proof Assume the condition holds. Then, by a theorem due to R. Doss [3, page 20] the space $\langle X, \tau\rangle$ has only one compatible uniformity. Hence if $\mathcal{U}_{\tau}$ is any compatible uniformity on $X$ and $\mathcal{U}_{\tau}^{\prime}$ is its anchored dual then $\mathcal{U}_{\tau}=\mathcal{U}_{\tau}^{\prime}$, which implies that $\mathcal{U}_{\tau}$ is PSPC.

4.2 Theorem Let $\langle X, \tau\rangle$ be a Hausdorff completely regular space. If $\langle X, \tau\rangle$ is locally compact and has a unique compactification then it has the PSPC property with respect to all of its compatible uniform structures.

Proof Recall that there is a one-to-one correspondence between the compatible uniformities on $\langle X, \tau\rangle$ that are precompact and its compactifications. Thus the condition that the space $\langle X, \tau\rangle$ has a unique compactification implies that $\langle X, \tau\rangle$ has a unique precompact uniform structure. This, in turn, is equivalent to the condition that $\langle X, \tau\rangle$ has a unique uniform structure (see Newns [9, Theorem 1]). Hence the space $\langle X, \tau\rangle$ has the PSPC property with respect to all of its compatible uniform structures.

4.3 Remark Each of the above two theorems provided a sufficient condition for all of the compatible uniformities of a completely regular space $\langle X, \tau\rangle$ to be PSPC. In case $\langle X, \tau\rangle$ is standard, these theorems provide sufficient conditions for all of the compatible uniformities of $\langle X, \tau\rangle$ to have the INH property.

Our next application is based on the following observation.

4.4 Theorem If a complete Hausdorff uniform space $\langle X, \mathcal{U}\rangle$ is PSPC then a subset $S$ of $X$ is relatively compact if and only if it is bounded.

Proof Assume that $\langle X, \mathcal{U}\rangle$ and $S$ are standard. If $B$ is bounded then $B \subseteq \operatorname{fin}(X)$. Since $\langle X, \mathcal{U}\rangle$ is PSPC, we have $\operatorname{fin}(X)=\operatorname{pns}(X)$; and since $\langle X, \mathcal{U}\rangle$ is complete, we have $\mathrm{ns}(X)=\operatorname{pns}(X)$. Hence $B \subseteq \mathrm{ns}(X)$, which implies that $B$ is relatively compact. The converse is obvious.

We need the following notation for the next theorem. Let $\langle X, \tau\rangle$ be a standard completely regular space, and let $\Lambda_{c}$ be the set of pseudo-metrics on $X$ that was described in Example 3.8. We shall use the symbol $\simeq_{c}$ to denote the uniformity on $X$ that is generated by $\Lambda_{c}$. Thus, given $x, y \in X$, we have

$$
x \simeq_{c} y \quad \text { if and only if } \quad f(x) \simeq f(y) \text { for all } f \in{ }^{\sigma} C(X) .
$$

Thus $y \in \mu_{c}(x)$ if and only if $|f(x)-f(y)|<\epsilon$ for each $f \in{ }^{\sigma} C(X)$ and each $\epsilon \in{ }^{\sigma} \mathbb{R}^{+}$. 
4.5 Theorem Let $\langle X, \tau\rangle$ be a completely regular space. If $\langle X, \tau\rangle$ is Lindelöf then the uniform space $\left\langle X, \Lambda_{c}\right\rangle$ is complete.

Proof Assume the data are standard. Recall that a standard uniform space is complete if and only if every pre-nearstandard point is nearstandard (see Luxemburg [7, Theorem 3.14.1, p. 78]). So fix $p \in \mathrm{pns}_{\Lambda_{c}}(X)$. We will show that $p$ is nearstandard. For each $f \in{ }^{\sigma} C(X)$ and each $m \in{ }^{\sigma} \mathbb{Z}^{+}$, let

$$
B_{f, m}=\left\{u \in X:\left|f(u)-{ }^{\circ} f(p)\right| \leq \frac{1}{m}\right\},
$$

where ${ }^{\circ} f(p)$ is the standard part of $f(p)$. Note that each $B_{f, m}$ is standard and contains $p$. Let $\mathcal{B}_{p}$ be the standard subset of $\mathcal{P}(X)$ whose standard elements consist of the sets $B_{f, m}$, and let $\mu\left(\mathcal{B}_{p}\right)$ be the monad of $\mathcal{B}_{p}$. Then $\mu_{c}(p)=\mu\left(\mathcal{B}_{p}\right)$. Therefore $\bigcap \mathcal{B}_{p} \subseteq \mu\left(\mathcal{B}_{p}\right)=\mu_{c}(p)$. Since the set $\cap \mathcal{B}_{p}$ is standard, if it is not empty, it contains a standard element $x$ with $p \simeq_{c} x$, proving that $p$ is a nearstandard element of $\left\langle X, \Lambda_{c}\right\rangle$. Thus the proof will be complete once we show that $\bigcap \mathcal{B}_{p} \neq \emptyset$. Note that $\mathcal{B}_{p}$ is a family of closed subsets of $X$. So the fact that $\langle X, \tau\rangle$ is Lindelöf will yield $\cap \mathcal{B}_{p} \neq \emptyset$ once it is established that $\mathcal{B}_{p}$ has the countable intersection property. For this, let $\left(C_{n}\right)$ be any standard countable subset of $\mathcal{B}_{p}$, which we may assume is decreasing. We will prove $\bigcap_{n \in \mathbb{Z}^{+}} C_{n} \neq \emptyset$ by showing that the condition $\bigcap_{n \in \mathbb{Z}^{+}} C_{n}=\emptyset$ will lead to a contradiction.

By the definition of $\mathcal{B}_{p}$, there is a standard sequence $\left(f_{n}\right)$ in $C(X)$ and a standard subsequence $\left(k_{n}\right)$ of the sequence $(m)$ with $C_{n}=B_{f_{n}, k_{n}}$ for each $n \in \mathbb{Z}^{+}$. Now, for each $n \in \mathbb{Z}^{+}$, let $g_{n}(u)=\inf _{v \in C_{n}}\left|f_{n}(u)-f_{n}(v)\right|$. Since $\left|g_{n}(x)-g_{n}(y)\right| \leq\left|f_{n}(x)-f_{n}(y)\right|$ for all $x, y \in X,{ }^{4}$ we have $g_{n} \in C(X)$. Let $g(u)=\sum_{n \in \mathbb{Z}^{+}} \min \left\{\left|g_{n}(u)\right|, 2^{-n}\right\}$. If $\bigcap_{n \in \mathbb{Z}^{+}} C_{n}=\emptyset$ then for each $u \in X$ there is an $n \in \mathbb{Z}^{+}$with $u \notin C_{n}$, which implies that $g_{n}(u) \neq 0$. Hence $g(u)>0$ for all $u \in X$, and $\frac{1}{g} \in{ }^{\sigma} C(X)$. Since $p \in C_{n}$ for all standard $n, g_{n}(p)=0$ for all standard $n$. Therefore $g_{\omega}(p)=0$ for all $n$ less than or equal to some unlimited $\omega \in \mathbb{Z}^{+}$. So we can write

$$
g(p)=\sum_{n \in \mathbb{Z}^{+}} \min \left\{\left|g_{n}(p)\right|, 2^{-n}\right\}=\sum_{n=\omega+1}^{\infty} \min \left\{\left|g_{n}(p)\right|, 2^{-n}\right\} \leq \frac{1}{2^{\omega}} .
$$

Hence $\frac{1}{g}(p) \geq 2^{\omega}$. This contradicts the fact that $p \in \operatorname{pns}_{\Lambda_{c}}(X)$.

\footnotetext{
${ }^{4}$ To see this inequality, fix $x, y \in X$ and $n \in \mathbb{Z}^{+}$. Then, for all $v \in C_{n}$, we can write $\left|f_{n}(x)-f_{n}(v)\right| \leq\left|f_{n}(y)-f_{n}(v)\right|+\left|f_{n}(x)-f_{n}(y)\right|$. Taking infimum over $v$ yields $g_{n}(x) \leq$ $g_{n}(y)+\left|f_{n}(x)-f_{n}(y)\right|$, or $g_{n}(x)-g_{n}(y) \leq\left|f_{n}(x)-f_{n}(y)\right|$. Similarly, $g_{n}(y)-g_{n}(x) \leq\left|f_{n}(x)-f_{n}(y)\right|$. These two inequalities yield $\left|g_{n}(x)-g_{n}(y)\right| \leq\left|f_{n}(x)-f_{n}(y)\right|$.
} 
4.6 Remark Recall that a completely regular space $\langle X, \tau\rangle$ is realcompact if and only if the uniform space $\left\langle X, \Lambda_{c}\right\rangle$ is complete. Thus the preceding discussion provides a nonstandard proof for the fact that a completely regular space that is Lindelöf is realcompact. The usual proofs of this theorem use the zero-set machinery developed within the theory of rings of continuous functions (see eg Gillman and Jerison [4]).

4.7 Theorem Let $\langle X, \tau\rangle$ be a $T_{0}$ space that is regular and Lindelöf, and let $S$ be a subset of $X$. Then $S$ is relatively compact if and only if it is topologically bounded.

Proof The forward direction is obvious. To prove the converse, note that since $S$ is topologically bounded, it is $\Lambda_{c}$-bounded as a subset of the uniform space $\left\langle X, \Lambda_{c}\right\rangle$. Moreover, $X$ is completely regular because it is regular and Lindelöf (see Pervin [11, Theorem 5.5.6, page 92 and Theorem 5.6.1, page 95]). In Example 3.8, we saw that the space $\left\langle X, \Lambda_{c}\right\rangle$ is PSPC and, by Theorem 4.5, it is complete. So, by Theorem 4.4, $S$ is relatively compact since it is a bounded subset of $\left\langle X, \Lambda_{c}\right\rangle$.

\section{References}

[1] N Bourbaki, Elements of mathematics. General topology. Part 1 and Part 2, Hermann, Paris; Addison-Wesley Publishing Co., Reading, Mass.-London-Don Mills, Ont. (1966)

[2] A Dickinson, Compactness conditions and uniform structures, Amer. J. Math. 75 (1953) 224-228

[3] R Doss, On uniform spaces with a unique structure, Amer. J. Math. 71 (1949) 19-23

[4] L Gillman, M Jerison, Rings of continuous functions, Springer-Verlag, New YorkHeidelberg (1976)Reprint of the 1960 edition, Graduate Texts in Mathematics, No. 43

[5] CW Henson, The monad system of the finest compatible uniform structure, Proc. Amer. Math. Soc. 51 (1975) 163-170

[6] C W Henson, L C Moore, Jr, Invariance of the nonstandard hulls of a uniform space, from: "Victoria Symposium on Nonstandard Analysis (Univ. Victoria, Victoria, B.C., 1972)", Springer, Berlin (1974) 85-98. Lecture Notes in Math., Vol. 369

[7] W A J Luxemburg, A general theory of monads, from: "Applications of Model Theory to Algebra, Analysis, and Probability (Inte rnat. Sympos., Pasadena, Calif., 1967)", Holt, Rinehart and Winston, New York (1969) 18-86

[8] E Nelson, Internal set theory: a new approach to nonstandard analysis, Bull. Amer. Math. Soc. 83 (1977) 1165-1198

[9] W F Newns, Uniform spaces with unique structure, Amer. J. Math. 79 (1957) 48-52 
[10] W Page, Topological uniform structures, Dover Publications, Inc., New York (1988)Revised reprint of the 1978 original

[11] W J Pervin, Foundations of general topology, Academic Press Textbooks in Mathematics, Academic Press, New York-London (1964)

[12] K D Stroyan, W A J Luxemburg, Introduction to the theory of infinitesimals, Academic Press [Harcourt Brace Jovanovich, Publishers], New York-London (1976)Pure and Applied Mathematics, No. 72

[13] N Vakil, Real analysis through modern infinitesimals, volume 140 of Encyclopedia of Mathematics and its Applications, Cambridge University Press, Cambridge (2011)

[14] N Vakil, R Vakil, A remark on uniform spaces with invariant nonstandard hulls, MLQ Math. Log. Q. 51 (2005) 610-612

[15] S Willard, General topology, Addison-Wesley Publishing Co., Reading, Mass.London-Don Mills, Ont. (1970)

Department Of Mathematics, Western Illinois University, Macomb, IL 61455

N-Vakil@wiu.edu

http://www.wiu.edu/users/mfnv/MySite/HomePage.html

Received: 3 March $2013 \quad$ Revised: 14 June 2014 